\title{
Teenagers' Usage of a Mobile-Wearable-Cloud Platform to Promote Healthy Lifestyles: the PEGASO Experience*
}

\author{
Maurizio Caon, Stefano Carrino, Leonardo Angelini, Omar Abou Khaled, Elena Mugellini, Filip \\ Velickovski, and Giuseppe Andreoni
}

\begin{abstract}
In contemporary society, non-communicable diseases linked to unhealthy lifestyles, such as obesity, are on the rise with a major impact on global deaths. Prevention is the new frontier, promising to increase life expectancy and quality, while reducing costs related to healthcare. The PEGASO project developed a mobile ecosystem where the digital Companion aims at empowering teenagers in the adoption of healthy lifestyles. The pilot study conducted in three European countries (Spain, UK and Italy) shows a good acceptance of the system and that teenagers are keen to use mobile technology to improve their lifestyle, although wearable devices did not engage the young users.
\end{abstract}

\section{INTRODUCTION}

Non-communicable diseases were responsible for $68 \%$ of global deaths in 2012, meaning that they caused 38 million of the world's 56 million deaths [1]. Unhealthy lifestyle behaviours such as diet, physical activity and sedentariness are key risk factors for major non-communicable diseases. Among these non-communicable diseases, obesity represents a major threat for global health in contemporary society. Several research studies demonstrate that juvenile obesity can lead to serious medical conditions and have important psychosocial consequences. Many lifestyle choices are made during adolescence, this makes this period a critical phase of personal development. In particular, behavioural patterns linked to physical activity, sleeping habits and nutrition are established during adolescence and people tend to maintain them throughout their whole lives [2]. Indeed, unhealthy diet, physical inactivity and sedentary behaviour are known to track from childhood into adulthood and are difficult to change later in life [3]. Hence, it is important to promote healthy lifestyle adoption during adolescence. This would allow having healthier communities, reducing risks linked to cardiovascular diseases, obesity and related comorbidities. A recent systematic review showed that there is an evidence that app-based interventions to improve diet, physical activity and sedentary behaviours can be effective [4]. Also, findings from other recent systematic reviews suggest that approaches based on mobile technology are feasible and acceptable tools

*Research supported by the European Commission under the 7th Framework Programme through the project grant $n^{\circ} 610727$.

M. Caon is with the University of Applied Sciences and Arts Western Switzerland, Fribourg, 1700 Switzerland (phone: +41264296748; e-mail: maurizio.caon@ hes-so.ch).

S. Carrino, L. Angelini, O. A. Khaled and E. Mugellini are with the University of Applied Sciences and Arts Western Switzerland, Fribourg, 1700 Switzerland.

F. Velickovski is with EURECAT, Carrer de Bilbao 72, 08005, Barcelona, Spain (e-mail: filip.velickovski@eurecat.org).

G. Andreoni is with the Politecnico di Milano, Sensibilab, Indaco Department, Via Durando 38/A, 20158, Milano, Italy (e-mail: giuseppe.andreoni@polimi.it). in particular for the prevention and treatment of paediatric obesity [5] and [6]

The EU-funded PEGASO project (Personalized guidance services for optimizing lifestyle in teen-agers through awareness, motivation and engagement) aims at developing a whole services ecosystem, which motivates teenagers to internalize healthy behaviours effortlessly to prevent diseases in adulthood and improve their quality of life. This multidisciplinary project enables the promotion of healthy lifestyles among teenagers through the use of assistive technology [7]. The core of this project is, indeed, represented by the ICT system, which allows provision of tailored interventions to encourage motivation towards healthy lifestyle habits to the teenage users via their smartphones [8].

Figure 1. Architecture overview of the PEGASO system.

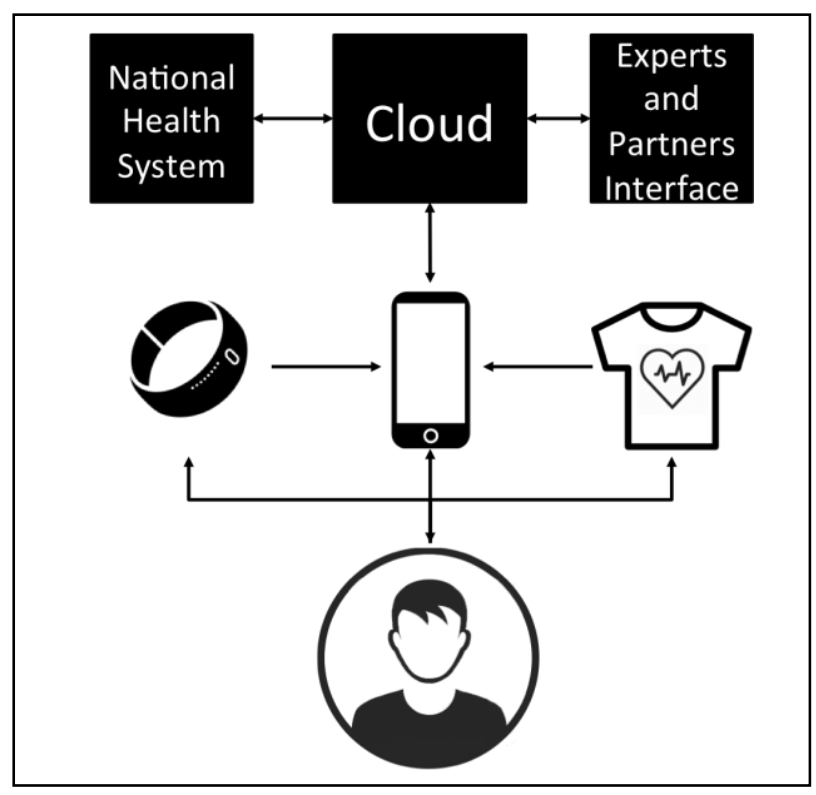

\section{THE PEGASO ECOSYSTEM}

Involving adolescents in participatory action for health promotion is gaining recognition as a viable approach not only to preventing youth problems, but also enhancing positive development [9]. As with other special populations, teenagers have peculiar needs with respect to healthcare [10]. Therefore, systems supporting health management for teenagers should be designed involving them in a co-creation process. In the PEGASO project, four stages of user-centred design were accomplished, during which several multitechnique workshops were organized with teenagers to elicit user requirements for the platform to promote healthy 
lifestyles. The first stage of workshops aimed at exploring the teenage perceptions about healthy living, the role of technology in health and its use for wellness promotion [10]. The second stage involved the adolescents in the interface codesign and evaluation of the first system mock-ups. The third stage involved the users in testing the developed prototypes and providing structured feedback to allow iterating on the prototype to improve the system and fix problems. The fourth stage had the teenagers testing the prototypes on a longer period of time and their feedback was used to enhance the experience and solve the last bugs.

The final version of the system was composed of the PEGASO Companion installed in the user's smartphone, connected to wearable devices to improve the user's behaviour monitoring and to the cloud for the personalisation of intervention and of the services (further details in [11]). The cloud could also securely transfer the user's data to the personal folder in the National Health System (NHS). Moreover, the PEGASO partners could access to the system via a specific interface like experts to answer questions raised by the users or to partners in order to allow provide and update services. The overview of the PEGASO system is depicted in Figure 1.

\section{A. The PEGASO Companion}

The PEGASO Companion vision is not linked to a unique physical device but it is conceived as a ubiquitous artificial intelligence, which changes form over time to accompany the user throughout her/his life adapting to her/his evolving needs and desires [12]. The PEGASO Companion is an electronic coach (eCoach) aiming at supporting the users in adopting healthy lifestyles. The development of eCoaches for health promotion is a current and growing trend. A recent scoping review analysed 32 eCoaches for healthy lifestyles showing that are able to influence positively both health outcomes and usability [13]. However, this literature review showed that there is no eCoach specially developed for teenagers. The actual implementation of the PEGASO Companion is embedded in the smartphone as a set of applications [14]. Every application is designed to deal with specific obesity risk factors (low physical activity, unhealthy nutrition or sedentary behaviour) or to deliver precise behaviour change interventions. The PEGASO ecosystem is modular, meaning that the user can choose what to install, and composed of the following apps:

- $\quad$ The Companion is the main access to the PEGASO ecosystem. This application allows adding friends, sends tailored messages, shows information to the user to coach and motivate.

- The eDiary is a mobile food record enables users to monitor their dietary behaviours while coaching the user with immediate educational feedback based on dietary data patterns introduced by the users.

- Challenges app motives the user to achieve their goals exploiting implementation intention and other social motivational elements.

- $\quad$ The PEGASO Serious Game proposes a full-fledged game aiming at engaging users while creating an opportunity for health literacy and providing behaviour-changing interventions.

\section{B. Sensors}

The PEGASO system bases the measurement of the user's behaviour mainly on the use of the sensors embedded in the smartphone, i.e., 3D inertial sensors, GPS and selfreported information. Since teenage users cannot always bring with them the smartphone (e.g., smartphones are forbidden in many schools and difficult to use in sports), the system was developed in order to include also two different wearable devices. The first is a smart bracelet named WWAT, to be worn all day and providing activity tracking and sleep monitoring. The second is a smart garment, to be worn especially during the physical activity to have a better analysis of this time and a more detailed vision of the physiological components characterizing the user during exercise. It is composed of a sensorised T-shirt or bra with embedded textile electrodes for measuring 1 ECG lead and a wearable device applied to the garments named WES embedding two additional 3D accelerometer and gyroscope placed at chest level approximately in correspondence of the 10 th rib. These wearable devices were specifically designed for and with adolescents [15]. Indeed, there are no commercial wearable devices designed to meet adolescents' needs and desires on both aesthetical and functional levels. For this reason, it was necessary to develop these specific wearables in the frame of the PEGASO project.

\section{The Cloud}

One of the key aspects of PEGASO is the personalisation of the interventions based on users' behaviours and preferences [16]. The data collection and computation of the tailoring algorithms are distributed between the smartphones and the cloud. Only the simple and fast computation that requires an immediate feedback is performed on the smartphones. However, all the data are always uploaded in the secured cloud as soon as the smartphone is connected to the Internet, where the algorithms for the tailoring of the intervention are executed. Such personalization is based on the development of a Virtual Individual Model (VIM) for characterizing the user on different aspects (i.e., physical, functional and behavioural parameters). The VIM is finally modelled through the implementation of an ad-hoc ontology [17] built upon a scoring system able to numerically represent the behavioural status of a user [18].

\section{Stakeholders Access}

The PEGASO system included a web interface that allowed researchers to control and modify some parameters of the study setting, to monitor the users' behaviours in an anonymous manner. Moreover, through this portal the teenagers could contact both the technical support service to help them troubleshooting in case of problem with their apps or devices, and ask questions to the physiology and nutrition experts present in the project consortium.

Another interface allowed the PEGASO partners to offer and update services for the users. The gamified approach used for the design of the PEGASO applications allows the user to collect FitCoins executing particular actions. These FitCoins can be used to buy real services provided through the "PEGASO City app", which creates the bridge that 
connects the digital world of the PEGASO system with the physical world. Examples of provided services can be a ride at the local bike sharing, one access to a local swimming pool, a healthy snack at a local cafeteria et cetera. This application plays a double role: first, it helps teenagers to find places that have agreements with PEGASO and that provide them opportunities for healthy behaviours. Second, it is a channel between the PEGASO project and possible partners.

\section{E. National Health System Connection}

The connection between the PEGASO ecosystem and the personal health folder is established through the BRIDGE system. The BRIDGE consists of the components allowing the extraction of some significant data collected and elaborated into the PEGASO ecosystem and to create a report to be shared with physicians into the personal health folder. The users could manage their own data and decide to share it with the physicians through a dedicated application called "Report App". The possibility to share the users' data concerning their lifestyle aimed at providing the physicians with further information in order to better inform their analysis of the users' health status.

\section{The PILOT STUDY}

\section{A. Study Design}

A pilot study was conducted aiming at assessing platform acceptability, usability and technical security, as well as, user satisfaction and preferences. The PEGASO system was tested in 4 different pilot sites (one in Italy, one in Spain and two in United Kingdom) where multiple schools accepted to participate in the trial. A total number of 368 teenagers were involved in the test. All of these teenagers created an account in the platform. The breakdown by country is the following: Italy: 99 teenagers, Spain: 119 teenagers, United Kingdom: England: 100 teenagers and Scotland: 50 teenagers. The data collection conducted during this pilot study lasted for 8 months and twenty days (from 1st October 2016 to 19th June 2017). The experimental procedures involving human subjects described in this paper were approved by the Institutional Review Boards in all the countries involved in the pilot study.

\section{B. Results and Discussion}

Figure 2 depicts the number of active users over the whole study period, although this was normalised meaning that the different study start dates of the pilot sites are all set on day 1 although they happened on different actual dates. The percentage of adoption, more than half of the population during the first two months, provides evidence that teenagers are keen to be engaged in using smartphones to improve their lifestyle. It is interesting also to note that teenagers rarely used the sensing platform, which was composed of the two wearable devices (the WWAT bracelet and the WES smart Tshirt). Figure 2 clearly shows how very few young students decided to use the wearable devices and in particular the sensing clothes, which were introduced on day 136. This outcome represents a very interesting and strong point to optimize a prevention intervention with teenagers: this kind of technologies is not a killer application for young people. Indeed, they do not use them: maybe for supervision fear or for the need to recharge frequently these devices (and this operation could be perceived as boring or even refused), in
Figure 2. Evolution of the usage of Companion and wearable devices (WWAT and WES) after their introduction at $136^{\text {th }}$ day of the pilot study.

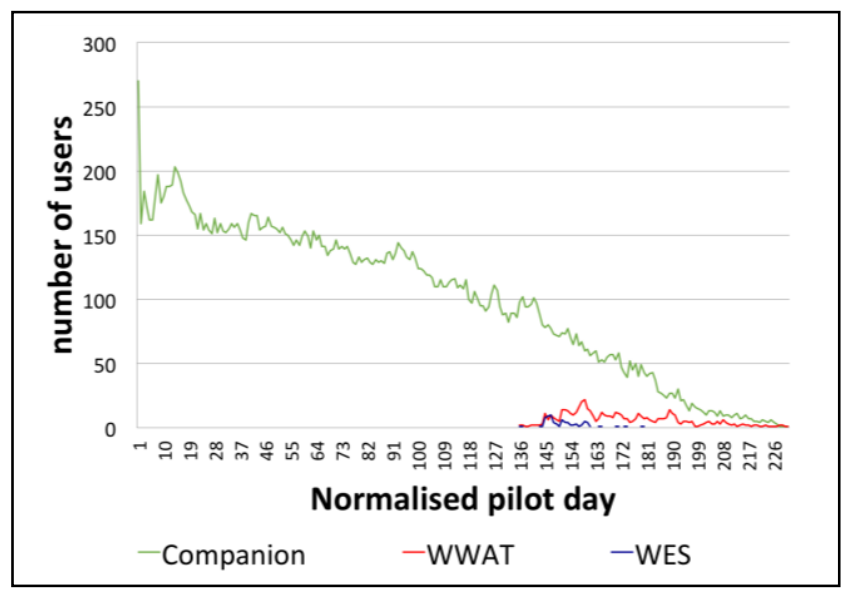

any case wearable systems are not specific for this target population. Only the smartphone is essential. These results are not surprising, indeed, during the first stage of the usercentred design process of the PEGASO project, teenagers already expressed their scepticism about the adoption of wearable devices for healthy lifestyles although they acknowledged their important potential [20]. Moreover, these results are confirmed by another recent study, in which the findings suggest that wearable devices designed to support healthy lifestyles may even have negative motivational consequences in teenage users [21]. In Figure 3, it is possible to analyse the use of the system with reference to the single user' activity. In this figure, only the access to the PEGASO Companion is reported, which was anyway the main gateway to access all the PEGASO services. It is interesting to note that the stripes of yellow points show that some teenagers were engaged on long periods of time, i.e., several weeks. This provides evidence that when an adolescent is interested in using a system for her health, she is committed to a sustained use over time, although rarely this engagement lasted for the whole 6-month period of the study. In addition to the previous measurements, the users evaluated the PEGASO Companion filling out two standard questionnaires: the System Usability Scale (SUS) [22] and the Emotional Metric Outcomes (EMO) [23]. The SUS is designed to measure user's subjective rating of a product's usability. The EMO is designed to assess the emotional outcomes of the interaction between users and a product. Both questionnaires were given to the adolescents towards the end of the pilot study, precisely at month 6 . The mean score for SUS for the PEGASO Companion was 64.66 out of 100 (standard deviation $=15.11)$. The percentage of participants that scored more than 68 points on the SUS (which is the average) was $40.9 \%(110 / 269)$. The mean score for the EMO questionnaire was 6.25 out of 10 (standard deviation $=1.41$ ) and 38 of 269 participants $(12.6 \%)$ scored 8 or more in the EMO questionnaire. These results show that the teenage users perceived the experience of use of the PEGASO system being average meaning that the interface is not particularly easy to use and with no great positive impact on their emotions. However, it is important to note that there is a group of users that evaluated the experience in a positive manner, which can be linked to those users that showed a long usage of the platform as depicted in Figure 3. 


\section{CONCLUSION}

This paper presents an overview of the PEGASO system and discusses the results of the pilot study conducted in four pilot sites in different European regions: Catalonia, Lombardy, England and Scotland. The results of the pilot study show that teenagers are keen to adopt smartphones as a tool to improve their diet, physical activity and sedentary behaviours in order to achieve a healthy lifestyle. On the other hand, wearable devices are not able to engage adolescents for the same purpose. This project shows that it is important to keep investing in further research for the development of mobile applications for the prevention of non-communicable diseases and for the direct empowerment of young users in order to involve them in a daily engagement for the promotion of their health and wellbeing.

Figure 3. Graphic representation of users' activity with the PEGASO Companion during the pilot study. The four pilot sites started the test on different dates and in this graph the test period has been normalized rendering all the pilot sites tests starting on day 0 .

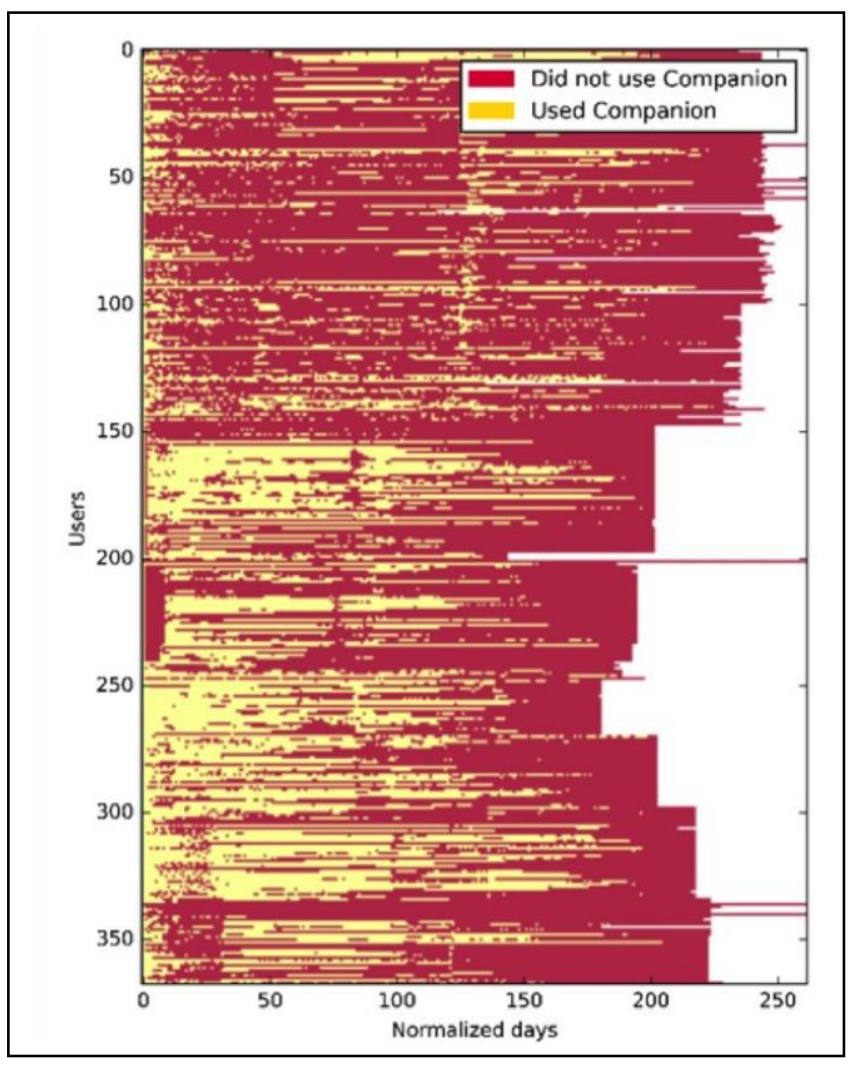

\section{REFERENCES}

[1] World Health Organization. Global status report on noncommunicable diseases. World Health Organization. 2014. http://www.who.int/nmh/ publications/ncd-status-report-2014/en/. Accessed 04/02/2018.

[2] Kumar, B., Robinson, R., \& Till, S. (2015). Physical activity and health in adolescence. Clinical Medicine, 15(3), 267-272.

[3] Craigie AM, Lake AA, Kelly SA, Adamson AJ, Mathers JC. Tracking of obesity-related behaviours from childhood to adulthood: a systematic review. Maturitas. 2011;70:266-84.

[4] Schoeppe S, Alley S, Van Lippevelde W, Bray NA, Williams SL, Duncan MJ, Vandelanotte C. Efficacy of interventions that use apps to improve diet, physical activity and sedentary behaviour: a systematic review. International Journal of Behavioral Nutrition and Physical Activity. 2016 Dec;13(1):127.
[5] Quelly SB, Norris AE, DiPietro JL. Impact of mobile apps to combat obesity in children and adolescents: A systematic literature review. Journal for Specialists in Pediatric Nursing. 2016 Jan 1;21(1):5-17.

[6] Turner T, Spruijt-Metz D, Wen CF, Hingle MD. Prevention and treatment of pediatric obesity using mobile and wireless technologies: a systematic review. Pediatric obesity. 2015 Dec 1;10(6):403-9.

[7] Guarneri R, Andreoni G. Active prevention by motivating and engaging teenagers in adopting healthier lifestyles. In Int. Conference on Digital Human Modeling and Applications in Health, Safety, Ergonomics and Risk Management 2014 (pp. 351-360). Springer.

[8] Carrino S, Caon M, Angelini L, ... \& Lafortuna C. PEGASO: A personalized and motivational ICT system to empower adolescents towards healthy lifestyles. Innovation in Medicine and Healthcare.

[9] Wong NT, Zimmerman MA, Parker EA. A typology of youth participation and empowerment for child and adolescent health promotion. American Journal of Community Psychology. 2010 Sep $1 ; 46(1-2): 100-14$.

[10] Lang AR, Craven MP, Atkinson S, Simons L, Cobb S, Mazzola M. Human factors multi-technique approach to teenage engagement in digital technologies health research. In Perspectives on HCI Research with Teenagers 2016 (pp. 61-101). Springer, Cham.

[11] Mazzola M, Arslan P, Cândea G, Radu C, Azzolini M, Degano C, Andreoni G. Integrated Architecture for Next-Generation m-Health Services (Education, Monitoring and Prevention) in Teenagers. InInternational Conference on Digital Human Modeling and Applications in Health, Safety, Ergonomics and Risk Management 2014 Jun 22 (pp. 403-414). Springer, Cham.

[12] Carrino S, Caon M, Khaled OA, Andreoni G, Mugellini E. PEGASO: Towards a life companion. InInternational Conference on Digital Human Modeling and Applications in Health, Safety, Ergonomics and Risk Management 2014 Jun 22 (pp. 325-331). Springer, Cham.

[13] Lentferink AJ, Oldenhuis HK, de Groot M, Polstra L, Velthuijsen H, van Gemert-Pijnen JE. Key Components in eHealth Interventions Combining Self-Tracking and Persuasive eCoaching to Promote a Healthier Lifestyle: A Scoping Review. Journal of medical Internet research. 2017 Aug;19(8).

[14] Caon M, Carrino S, Condon L, Ascolese A, Facchinetti S, Mazzola M, Perego P, Velickovski F, Andreoni G, Mugellini E. PEGASO Companion: A Mobile App to Promote Healthy Lifestyles Among Adolescents. In International Conference on Wireless Mobile Communication and Healthcare 2016 Nov 14 (pp. 53-61). Springer.

[15] Standoli CE, Guarneri MR, Perego P, Mazzola M, Mazzola A, Andreoni G. A Smart Wearable Sensor System for Counter-Fighting Overweight in Teenagers. Sensors. 2016 Aug 10;16(8):1220.

[16] Caon M, Carrino S, Lafortuna CL, Serrano JC, Coulson NS, Sacco M, Khaled OA, Mugellini E. Tailoring motivational mechanisms to engage teenagers in healthy life-style: A concept. In AHFE Conf. on Advances in Human Aspects of Healthcare 2014 Jul 19.

[17] Sojic, A., Terkaj, W., Contini, G., \& Sacco, M. (2016). Modularising ontology and designing inference patterns to personalise health condition assessment: the case of obesity. Journal of biomedical semantics, 7(1), 12.

[18] Velickovski F, Orte S, Sola M, Tabozzi SA, Lafortuna CL. Detection and Assessment of Behaviours Associated with the Risk of Obesity in Adolescents. IneHealth $360^{\circ} 2017$ (pp. 253-258). Springer, Cham.

[19] Adorni F, Prinelli F, Crespi C, ...\& Lang A. Personalised Guidance Services for Optimising Lifestyle in Teen-Agers Through Awareness, Motivation and Engagement-PEGASO: A Pilot Study Protocol. In International Conference on Wireless Mobile Communication and Healthcare 2016 Nov 14 (pp. 45-52). Springer.

[20] Carrion C, Caon M, Carrino S, ... \& Espallargues M. Wearable lifestyle tracking devices: are they useful for teenagers? In Proceedings of UbiComp 2015 (pp. 669-674). ACM.

[21] Kerner C, Goodyear VA. The motivational impact of wearable healthy lifestyle technologies. American Journal of Health Education. 2017 Sep 3;48(5):287-97.

[22] Bangor A, Kortum PT, Miller JT. An empirical evaluation of the system usability scale. Intl. Journal of Human-Computer Interaction. 2008 Jul 29;24(6):574-94.

[23] Lewis JR, Mayes DK. Development and psychometric evaluation of the Emotional Metric Outcomes (EMO) questionnaire. International Journal of Human-Computer Interaction. 2014 Sep 2;30(9):685-702. 